\title{
Volterra Type Integral Operators and Composition Operators on Model Spaces
}

\author{
Tesfa Mengestie \\ Department of Mathematical Sciences, Stord/Haugesund University College (HSH), Klingenbergvegen 8, 5414 Stord, Norway \\ Correspondence should be addressed to Tesfa Mengestie; tesfantnu@gmail.com
}

Received 16 October 2014; Accepted 6 January 2015

Academic Editor: Ruhan Zhao

Copyright (C) 2015 Tesfa Mengestie. This is an open access article distributed under the Creative Commons Attribution License, which permits unrestricted use, distribution, and reproduction in any medium, provided the original work is properly cited.

\begin{abstract}
We study some mapping properties of Volterra type integral operators and composition operators on model spaces. We also discuss and give out a couple of interesting open problems in model spaces where any possible solution of the problems can be used to study a number of other operator theoretic related problems in the spaces.
\end{abstract}

\section{Introduction}

We denote by $\mathscr{H}$ the space of holomorphic functions. We define the Volterra type integral operator on $\mathscr{H}$ induced by a homomorphic symbol $g$ by

$$
V_{g} f(z)=\int_{0}^{z} f(w) g^{\prime}(w) d w .
$$

Questions about various operator theoretic properties of $V_{g}$ expressed in terms of function theoretic conditions on $g$ have been a subject of high interest mainly after the works of Pommerenke [1] and later by Aleman et al. [2-4]. In [3], it was proved that $V_{g}$ on the Hardy space $H^{p}(\mathbb{D}), 1 \leq p<\infty$, is bounded if and only if $g$ belongs to the space of BMOA, while compactness is characterized in terms of the corresponding VMOA spaces.

In this paper we plan to study the bounded, compact, and Hilbert-Schmidt properties of the Volterra type integral operators and composition operators acting between the model spaces $K_{I}^{2}$ and the Hardy space $H^{2}\left(\mathbb{C}_{+}\right)$of the upperhalf plane. We recall that the model spaces $K_{I}^{2}$, where $I$ is an inner function, are the subspace of $H^{2}\left(\mathbb{C}_{+}\right)$defined by $K_{I}^{2}=H^{2}\left(\mathbb{C}_{+}\right) \ominus I H^{2}\left(\mathbb{C}_{+}\right)$. The spaces $K_{I}^{2}$ are reproducing kernel Hilbert spaces with kernel function

$$
K_{w}(z)=\frac{i}{2 \pi} \cdot \frac{1-\overline{I(w)} I(z)}{z-\bar{w}}, \quad k_{w}(z)=\frac{K_{w}(z)}{\left\|K_{w}\right\|_{2}} .
$$

Each inner function $I$ has either one or infinitely many components. We call it one-component if there exists a $\delta$ in $(0,1)$ for which the level set $\left\{z \in \mathbb{C}_{+}:|I(z)|<\delta\right\}$ is connected. For model spaces generated by such class of inner functions, we prove the following.

Theorem 1. Let I be a one-component inner function on $\mathbb{C}_{+}$ and let $g$ be a holomorphic function on $\mathbb{C}_{+}$. Then $V_{g}: K_{I}^{2} \rightarrow$ $H^{2}\left(\mathbb{C}_{+}\right)$is

(i) bounded if and only if

$$
\sup _{w \in \mathbb{C}_{+}} \int_{\mathbb{C}_{+}}\left|k_{w}(z)\right|^{2}\left|g^{\prime}(z)\right|^{2} \Im z d A(z)<\infty ;
$$

(ii) compact if and only if

$$
\lim _{|w| \rightarrow \infty} \int_{\mathbb{C}_{+}}\left|k_{w}(z)\right|^{2}\left|g^{\prime}(z)\right|^{2} \Im z d A(z)=0 ;
$$

(iii) in the Hilbert-Schmidt class $\mathcal{S}_{2}\left(K_{I}^{2}, H^{2}\left(\mathbb{C}_{+}\right)\right)$if and only if

$$
\int_{\mathbb{C}_{+}}\left|g^{\prime}(z)\right|^{2}\left(1-|I(z)|^{2}\right) d A(z)<\infty
$$

where $d A$ denotes the usual Lebesgue area measure. 
The criteria found above classify the bounded and compact $V_{g}$ on model spaces in terms of their action on the reproducing kernels. As will be seen in Section 4, by converting the boundary integral in Hardy spaces to an area integral in terms of derivatives, we could reduce the boundedness and compactness problems of $V_{g}$ into questions of bounded and compact Carleson embedding maps (measures) for $K_{I}^{2}$. Such measures have been identified when $I$ belongs to the class of one-component inner functions; for example, see [5-10]. Despite several attempts made by many experts in the subject, a complete identification of the measures remains open when $I$ belongs to the class of infinitely many component inner functions. For a special class of model spaces which admit reproducing kernel Riesz bases corresponding to sparse sequence of points, which includes the de Branges spaces, the author together with Belov et al. [11] has described the measures in terms of a condition analogous to the $A_{2}$ weight condition. In what follows we will use those results in [11] to study the bounded and compact properties of $V_{g}$. We note in passing that those spaces constitute model spaces generated by the class of infinitely many component inner functions.

1.1. The Volterra Type Integral Operators on $\mathscr{H}(\Gamma, v)$. Let $\Gamma=$ $\left(\gamma_{n}\right)$ be a sequence of distinct complex numbers and let $v=\left(v_{n}\right)$ be a weight sequence that satisfies the admissibility condition

$$
\sum_{n=1}^{\infty} \frac{v_{n}}{1+\left|\gamma_{n}\right|^{2}}<\infty
$$

Any such pair $(\Gamma, v)$ parameterizes the space $\mathscr{H}(\Gamma, v)$ which consists of all functions

$$
f(z)=\sum_{n=1}^{\infty} \frac{a_{n} v_{n}}{z-\gamma_{n}}
$$

for which

$$
\begin{aligned}
& \|f\|_{\mathscr{H}(\Gamma, v)}=\left\|\left(a_{n}\right)\right\|_{\ell_{v}^{2}}<\infty, \\
& \ell_{v}^{2}=\left\{\left(a_{n}\right):\|a\|_{v}^{2}=\sum_{n=1}^{\infty}\left|a_{n}\right|^{2} v_{n}<\infty\right\}
\end{aligned}
$$

and $z$ belongs to the set

$$
(\Gamma, v)^{*}=\left\{z \in \mathbb{C}: \sum_{n=1}^{\infty} \frac{v_{n}}{\left|z-\gamma_{n}\right|^{2}}<\infty\right\} .
$$

The equation in (7) means that we obtain the value of a function $f$ in $\mathscr{H}(\Gamma, v)$ at a point $z$ in $(\Gamma, v)^{*}$ by computing the weighted discrete Hilbert transform:

$$
\left(a_{n}\right) \longmapsto \sum_{n=1}^{\infty} \frac{a_{n} v_{n}}{z-\gamma_{n}}
$$

which is well defined whenever $\left(a_{n}\right)$ belongs to $\ell_{v}^{2}$. This follows from an application of Cauchy-Schwarz inequality along with (8) and (9). The de Branges spaces, model subspaces of the Hardy space $H^{2}$ which admit Riesz bases of reproducing kernels, and the Fock-type spaces studied in [12] are all examples of spaces of the kind $\mathscr{H}(\Gamma, v)$. We refer to $[11,13,14]$ for detailed accounts. The Carleson measures on $\mathscr{H}(\Gamma, v)$ have been described in [11] when $\Gamma$ grows at least exponentially, that is, when

$$
\inf _{n \geq 1} \frac{\left|\gamma_{n+1}\right|}{\left|\gamma_{n}\right|}>1
$$

When we consider such sparse sequence $\Gamma$, it is natural to partition $\mathbb{C}_{+}$in the following way. We set $\Omega_{1}=\left\{z \in \mathbb{C}_{+}\right.$: $\left.|z|<\left(\left|\gamma_{1}\right|+\left|\gamma_{2}\right|\right) / 2\right\}$ and then for $n \geq 2$

$$
\Omega_{n}=\left\{z \in \mathbb{C}_{+}: \frac{\left(\left|\gamma_{n-1}\right|+\left|\gamma_{n}\right|\right)}{2} \leq|z|<\frac{\left(\left|\gamma_{n}\right|+\left|\gamma_{n+1}\right|\right)}{2}\right\} .
$$

We now state our first result on $\mathscr{H}(\Gamma, v)$.

Theorem 2. Suppose that the sequence $\Gamma$ satisfies the sparseness condition (11) and $g:(\Gamma, v)^{*} \rightarrow \mathbb{C}_{+}$is homomorphic. Then $V_{g}: \mathscr{H}(\Gamma, v) \rightarrow H^{2}\left(\mathbb{C}_{+}\right)$is

(i) bounded if and only if

$$
\begin{gathered}
\sup _{n \geq 1} \int_{\Omega_{n}} \frac{v_{n}\left|g^{\prime}(z)\right|^{2} \Im z d A(z)}{\left|z-\gamma_{n}\right|^{2}}<\infty, \\
\sup _{n \geq 1}\left(\sum_{l=1}^{n} v_{l} \sum_{m=n+1}^{\infty} \int_{\Omega_{m}} \frac{\left|g^{\prime}(z)\right|^{2} \Im z d A(z)}{|z|^{2}}\right. \\
\left.+\sum_{l=n+1}^{\infty} \frac{v_{l}}{\left|\gamma_{l}\right|^{2}} \sum_{m=1}^{n} \int_{\Omega_{m}}\left|g^{\prime}(z)\right|^{2} \Im z d A(z)\right)<\infty ;
\end{gathered}
$$

(ii) compact if and only if the "little oh" counterparts of (13) and (14) hold;

(iii) in the Hilbert-Schmidt class $\mathcal{S}_{2}\left(\mathscr{H}(\Gamma, v), H^{2}\left(\mathbb{C}_{+}\right)\right)$if and only if

$$
\sum_{n=1}^{\infty} \int_{\Omega_{n}} \frac{v_{n}\left|g^{\prime}(z)\right|^{2} \Im z}{\left|z-\gamma_{n}\right|^{2}} d A(z)<\infty
$$

$$
\begin{aligned}
\sum_{k=1}^{\infty} v_{k} & \sum_{k=n+1}^{\infty} \int_{\Omega_{k}} \frac{\left|g^{\prime}(z)\right|^{2} \Im z d A(z)}{|z|^{2}} \\
& +\sum_{k=n+1}^{\infty} \frac{v_{n}}{\left|\gamma_{n}\right|^{2}} \sum_{k=1}^{n} \int_{\Omega_{n}}\left|g^{\prime}(z)\right|^{2} \Im z d A(z)<\infty .
\end{aligned}
$$

Condition (13) of the theorem is a condition about the local behavior of the symbol $g$, while condition (14) deals with its global behavior. Combining the two conditions, we see that (13) may be replaced by the stronger global condition

$$
\sup _{n \geq 1} \int_{\mathbb{C}_{+}} \frac{v_{n}\left|g^{\prime}(z)\right|^{2} \mathfrak{\Im} z d A(z)}{\left|z-\gamma_{n}\right|^{2}}<\infty .
$$


In some special cases, this condition or the original one (13) automatically gives condition (14), for example, if the weight sequence $v_{n}$ grows at least exponentially and the numbers $v_{n} /\left|\gamma_{n}\right|^{2}$ decay at least exponentially with $n$. The same conclusion follows if the weight sequence $v_{n}$ is also summable.

\section{The Composition Operator on $\mathscr{H}(\Gamma, v)$}

Let $\psi$ be a homomorphic function in a given domain. We denote by $C_{\psi}$ the composition operator $f \mapsto f \circ \psi$. As a consequence of Littlewood's Subordination Theorem [15], it has long been known that all composition operators are bounded on all the Hardy spaces $H^{p}$ of the unit disc, where $0<p \leq \infty$. Although corresponding Hardy spaces of the disc and half-plane are isomorphic, composition operators act differently in the two domains. Unlike the case of $H^{p}(\mathbb{D})$, not all composition operators are bounded on $H^{p}\left(\mathbb{C}_{+}\right)$.

The study of compact composition operators on $H^{2}(\mathbb{D})$ first appeared in Schwartz [16] thesis in the late sixties though a complete function theoretic characterization, in terms of the inducing map's Nevanlinna counting function, was obtained by Shapiro later in [17]. Unlike $H^{p}(\mathbb{D})$ again, it was proved in [18] that there exists no compact composition operator on the Hardy space $H^{p}\left(\mathbb{C}_{+}\right)$. The work in [17] was continued by Lyubarskii and Malinnikova [19] for $C_{\psi}: K_{I}^{2}(\mathbb{D}) \rightarrow$ $H^{2}(\mathbb{D})$ and a complete extension was made when the generating inner function $I$ is one-component. More general trace ideal criteria for $C_{\psi}$ were obtained later in [20]. The problem remains open when $I$ has infinitely many components.

In this section, we study the bounded, compact, and Hilbert-Schmidt properties of composition operators on model spaces when the spaces admit normalized reproducing kernel Riesz bases associated with sparse sequences of points in $\mathbb{C}_{+}$. We note that such spaces represent some classes of model spaces generated by infinitely many component inner functions.

Theorem 3. Suppose that the sequence $\Gamma$ satisfies the sparseness condition (11) and $\psi$ is a nonconstant analytic function on $\mathbb{C}_{+}$, and $m$ refers to the usual Lebesgue measure on the real line. Then $C_{\psi}: \mathscr{H}(\Gamma, v) \rightarrow H^{2}\left(\mathbb{C}_{+}\right)$is

(i) bounded if and only if

$$
\begin{gathered}
\sup _{n \geq 1} \sup _{y \geq 0} \int_{\psi\left(\Omega_{n}\right)} \frac{v_{n} d x}{\left|\psi(x+i y)-\gamma_{n}\right|^{2}}<\infty, \\
\sup _{n \geq 1}\left(\sum_{l=1}^{n} v_{l} \sum_{k=n+1}^{\infty} \sup _{y \geq 0} \int_{\psi\left(\Omega_{k}\right)} \frac{d x}{|\psi(x+i y)|^{2}}\right. \\
\left.+\sum_{l=n+1}^{\infty} \frac{v_{l}}{\left|\gamma_{l}\right|^{2}} \sup _{y \geq 0} \sum_{k=1}^{n} m\left(\psi\left(\Omega_{k}\right)\right)\right)<\infty,
\end{gathered}
$$

where $\psi\left(\Omega_{k}\right)=\left\{\psi(x+i y): x+i y \in \Omega_{k}\right\}$;

(ii) compact if and only if the "little oh" counterparts of conditions (17) hold; (iii) in the Hilbert-Schmidt class $\delta_{2}\left(\mathscr{H}(\Gamma, v), H^{2}\left(\mathbb{C}_{+}\right)\right)$if and only if

$$
\begin{aligned}
& \sum_{k=1}^{\infty} \sup _{y>0} \int_{\psi\left(\Omega_{k}\right)} \frac{v_{k} d x}{\left|\psi(x+i y)-\gamma_{k}\right|^{2}}<\infty, \\
& \sum_{k=1}^{\infty} v_{k} \sum_{j=k+1}^{\infty} \sup _{y>0} \int_{\psi\left(\Omega_{j}\right)} \frac{d x}{|\psi(x+i y)|^{2}} \\
& \quad+\sum_{k=1}^{\infty} \frac{v_{k}}{\left|\gamma_{k}\right|^{2}} \sup _{y>0} \sum_{j=1}^{k-1} m\left(\psi\left(\Omega_{j}\right)\right)<\infty .
\end{aligned}
$$

\section{Open Problems}

3.1. Open Problem 1. As will be seen in the next section, the proof of our boundedness and compactness results for both $C_{\psi}$ and $V_{g}$ relies mainly on previously obtained Carleson measure results on $\mathscr{H}(\Gamma, v)$. When the sequence of points $\gamma_{n}=n$ and the weight sequences $v_{n} \simeq 1$, the space $\mathscr{H}(\Gamma, v)$ becomes the classical Paley-Wiener space for which its Carleson measures have long been identified. When the sequence $\gamma_{n}$ grows at least exponentially, the measures are completely described in terms of $A_{2}$ type condition in [11]. The case when $\gamma_{n}$ grows between these two extreme cases remains an interesting open problem. A solution of this will settle the long-standing open problem of identifying the Carleson measures for model spaces, of course modulo the existence of reproducing kernel Riesz basis in model spaces. The novelty and the core of the approach in [11] has been in turning a number of problems including the Carleson measure problem into questions about different mapping properties of Hilbert transforms in weighted spaces of functions and sequences. To extend the approach, one may need to look into all possible interplays between the smoothness (regularity) of the weight sequence $\left(v_{n}\right)$ and the growth of the sequence $\Gamma=\left(\gamma_{n}\right)$.

3.2. Open Problem 2. Both of our results in parts (iii) of Theorems 2 and 3 deal with the Hilbert-Schmidt properties of the operators $V_{g}$ and $C_{\psi}$ on $\mathscr{H}(\Gamma, v)$. A more natural and interesting question is to ask the Schatten $\mathcal{S}_{p}$ class membership of these maps from $\mathscr{H}(\Gamma, v)$ to $H^{2}\left(\mathbb{C}_{+}\right)$for all $p$ in the range $0<p<\infty$. Dealing with these problems would have been easier had we known a complete characterization of the $\delta_{p}$ class membership of the imbedding maps induced by Carleson measures for the spaces, which itself is still another open problem. A good starting point for all of these problems could be to consider first the case when sequence $\gamma_{n}$ grows at least exponentially. A very special case of the $\delta_{p}$ membership problem pertaining to the embedding maps may be seen in [21].

A word on notation: the notation $U(z) \lesssim V(z)$ (or equivalently $V(z) \gtrsim U(z)$ ) means that there is a constant $C$ such that $U(z) \leq C V(z)$ holds for all $z$ 's in the set of a question. We write $U(z) \simeq V(z)$ if both $U(z) \lesssim V(z)$ and $V(z) \lesssim U(z)$. 


\section{Proofs of the Results}

Proof of Theorem 1. The key tool in the proofs of the first two results is the Littlewood-Paley description of Hardy spaces in terms of derivative

$$
\|f\|_{2}^{2}=2 \int_{\mathbb{C}_{+}}\left|f^{\prime}(z)\right|^{2} \Im z d A(z)
$$

for each $f$ in $H^{2}\left(\mathbb{C}_{+}\right)$. The formula helps to convert a boundary integral to an area integral in terms of derivatives. Since $\left(V_{g} f\right)^{\prime}(z)=f(z) g^{\prime}(z)$, an application of the above identity ensures that for each $f$ in $K_{I}^{2}$ we have

$$
\left\|V_{g} f\right\|_{2}^{2}=2 \int_{\mathbb{C}_{+}}|f(z)|^{2}\left|g^{\prime}(z)\right|^{2} \Im z d A(z) .
$$

It follows from this that the boundedness of the operator $V_{g}$ : $K_{I}^{2} \rightarrow H^{2}\left(\mathbb{C}_{+}\right)$is equivalent to saying that the measure $\mu_{g}$ where

$$
d \mu_{g}(z)=\left|g^{\prime}(z)\right|^{2} \Im z d A(z)
$$

is a Carleson measure for $K_{I}^{2}$. Assuming that $I$ is a onecomponent inner function, a result of Cohn [8] ensures that the measure property holds if and only if the normalized reproducing kernels of $K_{I}^{2}$ are uniformly embedded into $L^{2}\left(\mathbb{C}_{+}, d \mu_{g}\right)$ from which the boundedness part of the result follows.

To prove (ii), first from a standard normal family argument, we have that $V_{g}$ is compact if and only if $V_{g} f_{n} \rightarrow 0$ in $H^{2}\left(\mathbb{C}_{+}\right)$for each sequence of functions $f_{n}$ in $K_{I}^{2}$ such that the sequence is uniformly bounded in norm and converges uniformly to zero on compact subsets of $\mathbb{C}_{+}$. It means that the compactness of $V_{g}$ is equivalent to saying that the embedding map from $K_{I}^{2}$ to $L^{2}\left(\mu_{g}\right)$ is compact. Since $I$ is one-component, then the desired conclusion follows from a result of Cohn [8] again.

To prove part (iii), recall that the operator $V_{g}$ belongs to the Hilbert-Schmidt class if and only if, for any orthonormal basis $\left(e_{n}\right)$ of $K_{I}^{2}$, the sequence $\left(\left\|V_{g} e_{n}\right\|_{2}^{2}\right)$ is summable. It follows by (20) that

$$
\begin{aligned}
\sum_{n=1}^{\infty}\left\|V_{g} e_{n}\right\|_{2}^{2} & \simeq \sum_{n=1}^{\infty} \int_{\mathbb{C}_{+}}\left|e_{n}(z)\right|^{2}\left|g^{\prime}(z)\right|^{2} \Im z d A(z) \\
& =\int_{\mathbb{C}_{+}} \sum_{n=1}^{\infty}\left|e_{n}(z)\right|^{2}\left|g^{\prime}(z)\right|^{2} \Im z d A(z)
\end{aligned}
$$

On the other hand, because of the reproducing property of the kernel and Parseval's identity, we have

$$
\begin{gathered}
K_{w}(z)=\sum_{n=1}^{\infty}\left\langle K_{w}, e_{n}\right\rangle e_{n}(z)=\sum_{n=1}^{\infty} e_{n}(z) \overline{e_{n}(w)}, \\
\left\|K_{w}\right\|_{2}^{2}=\sum_{n=1}^{\infty}\left|e_{n}(w)\right|^{2} .
\end{gathered}
$$

The desired conclusion easily follows since

$$
\sum_{n=1}^{\infty}\left|e_{n}(z)\right|^{2}=\left\|K_{z}\right\|_{2}^{2} \simeq \frac{1-|I(z)|^{2}}{\Im} z
$$

Proof of Theorem 2. Applying the Littlewood-Paley identity again, the estimate

$$
\left\|V_{g} f\right\|_{2}^{2}=2 \int_{\mathbb{C}_{+}}|f(z)|^{2}\left|g^{\prime}(z)\right|^{2} \Im z d A(z) \lesssim\|f\|_{\mathscr{H}(\Gamma, v)}^{2}
$$

holds if and only if the embedding map from $\mathscr{H}(\Gamma, v)$ to $L^{2}\left(\mathbb{C}, \mu_{g}\right)$ is bounded, where $\mu_{g}$ is the nonnegative measure defined by (21). Then, the boundedness part of the result immediately follows from an application of Theorem 1.1 in [11].

By a result of Nordgren's [22], a sequence of functions $f_{n}$ in $\mathscr{H}(\Gamma, v)$ converges weekly to zero if and only if it converges pointwise to zero and $\sup _{n}\left\|f_{n}\right\|_{\mathscr{H}(\Gamma, v)}<\infty$. It follows that $V_{\psi}$ is compact if and only if the measure $\mu_{g}$ is a vanishing Carleson measure for $\mathscr{H}(\Gamma, v)$. Then an application of Theorem 1.2 in [14] finishes the proof of the compactness part.

It remains to prove the statement about Hilbert-Schmidt membership. First, we may observe that the reproducing kernel of $\mathscr{H}(\Gamma, v)$ at a point $\lambda$ in $(\Gamma, v)^{*}$ is explicitly given by

$$
k_{\lambda}(z)=\sum_{n=1}^{\infty} \frac{v_{n}}{\left(\bar{\lambda}-\overline{\gamma_{n}}\right)\left(z-\gamma_{n}\right)} ;
$$

this is a direct consequence of the definition of the space $\mathscr{H}(\Gamma, v)$. Furthermore, the sequence $e_{n}(z)=\sqrt{v_{n}} /\left(z-\gamma_{n}\right)$ constitutes an orthonormal basis to $\mathscr{H}(\Gamma, v)$. Then

$$
\begin{aligned}
\left\|V_{g}\right\|_{\mathcal{S}_{2}}^{2} & =\sum_{n=1}^{\infty}\left\|V_{g} e_{n}\right\|_{2}^{2} \simeq \sum_{n=1}^{\infty} \int_{\mathbb{C}_{+}}\left|e_{n}(z)\right|^{2}\left|g^{\prime}(z)\right|^{2} \Im z d A(z) \\
& =\int_{\mathbb{C}_{+}}\left\|K_{z}\right\|_{\mathscr{H}(\Gamma, v)}^{2}\left|g^{\prime}(z)\right|^{2} \Im z d A(z) \\
& =\int_{\mathbb{C}_{+}} \sum_{n=1}^{\infty} \frac{v_{n}}{\left|z-\gamma_{n}\right|^{2}}\left|g^{\prime}(z)\right|^{2} \Im z d A(z)
\end{aligned}
$$

Using the growth condition (11), we observe that if $z \in \Omega_{m}$, then

$$
\sum_{n=1}^{\infty} \frac{v_{n}}{\left|z-\gamma_{n}\right|^{2}} \simeq \sum_{n=1}^{m-1} \frac{v_{n}}{|z|^{2}}+\frac{v_{m}}{\left|z-\gamma_{m}\right|^{2}}+\sum_{n=m+1}^{\infty} \frac{v_{n}}{\left|\gamma_{n}\right|^{2}} .
$$

Plugging this estimate in (27), we find that $\left\|V_{g}\right\|_{\mathcal{S}_{2}}$ is finite if and only if both equations of (15) hold.

Proof of Theorem 3. Suppose now that $\psi$ is analytic on $\mathbb{C}_{+}$. Then, for each fixed $y>0$, we define $\psi_{y}(x)=\psi(x+i y)$ from $\mathbb{R}$ to $\mathbb{C}_{+}$. We denote by $m \psi_{y}^{-1}$ the pullback measure

$$
m \psi_{y}^{-1}(E)=m\left(\psi_{y}^{-1}(E)\right)
$$


for each Borel subset $E \subset \mathbb{C}_{+}$, where $m$ refers to the usual Lebesgue measure on the real line.

Applying change of variables, we observe

$$
\int_{\mathbb{R}}\left|C_{\psi} f(x+i y)\right|^{2} d x=\int_{\mathbb{C}_{+}}|f(z)|^{2} d m \psi_{y}^{-1}(z),
$$

which holds because of a classical result in measure theory ([23], page 163). This implies that the estimate

$$
\left\|C_{\psi} f\right\|_{2}^{2}=\sup _{y>0} \int_{\mathbb{C}_{+}}|(f \circ \psi)(x+i y)|^{2} d x \leqslant\|f\|_{\mathscr{H}(\Gamma, v)}^{2}
$$

holds if and only if $\left(m \psi_{y}^{-1}\right)_{y>0}$ constitute a family of Carleson measures for $\mathscr{H}(\Gamma, v)$ with a uniform bound on $y$ in the sense that

$$
\sup _{y>0} \frac{\int_{\mathbb{C}_{+}}|f(z)|^{2} d m \psi_{y}^{-1}(z)}{\|f\|_{\mathscr{H}(\Gamma, v)}^{2}}<\infty
$$

Then an application of Theorem 1.1 of [11] gives the first part of the result in the theorem.

The compactness part of our result follows easily by similar arguments used to prove the compactness part in Theorem 2 along with Theorem 1.2 of [14]. Thus, we remain to prove part (iii) of the result. Proceeding as in the proof of part (iii) of Theorem 2 and applying (31) we have

$$
\begin{aligned}
\left\|C_{\psi}\right\|_{\mathcal{S}_{2}}^{2} & =\sum_{n=1}^{\infty}\left\|C_{\psi} e_{n}\right\|_{2}^{2}=\sum_{n=1}^{\infty} \sup _{y>0} \int_{\mathbb{C}_{+}}\left|\left(e_{n} \circ \psi\right)(x+i y)\right|^{2} d x \\
& =\sum_{n=1}^{\infty} \sup _{y>0} \int_{\mathbb{C}_{+}}\left|e_{n}(z)\right|^{2} d m \psi_{y}^{-1}(z) \\
& =\sum_{n=1}^{\infty} \sup _{y>0} \int_{\mathbb{C}_{+}} \frac{v_{n}}{\left|z-\gamma_{n}\right|^{2}} d m \psi_{y}^{-1}(z) \\
& =\sum_{n=1}^{\infty} \sup _{y>0} \sum_{j=1}^{\infty} \int_{\Omega_{j}} \frac{v_{n}}{\left|z-\gamma_{n}\right|^{2}} d m \psi_{y}^{-1}(z) .
\end{aligned}
$$

Applying the growth condition in (11), we observe that if $z \in$ $\Omega_{n}$, then

$$
\begin{aligned}
& \sum_{n=1}^{\infty} v_{n} \sup _{y>0} \sum_{j=1}^{\infty} \int_{\Omega_{j}} \frac{d m \psi_{y}^{-1}(z)}{\left|z-\gamma_{n}\right|^{2}} \simeq \sum_{n=1}^{\infty} v_{n} \sup _{y>0} \sum_{j=1}^{n-1} \int_{\Omega_{j}} \frac{d m \psi_{y}^{-1}(z)}{|z|^{2}} \\
& +\sum_{n=1}^{\infty} \sup _{y>0}\left(\int_{\Omega_{n}} \frac{v_{n} d m \psi_{y}^{-1}(z)}{\left|z-\gamma_{n}\right|^{2}}+\sum_{j=n+1}^{\infty} v_{n} \int_{\Omega_{j}} \frac{d m \psi_{y}^{-1}(z)}{\left|\gamma_{n}\right|^{2}}\right) .
\end{aligned}
$$

The sum on the right-hand side of (34) can be further rewritten as

$$
\begin{aligned}
\sum_{n=1}^{\infty} v_{n} \sup _{y>0} \sum_{j=1}^{n-1} \int_{\Omega_{j}} \frac{d m \psi_{y}^{-1}(z)}{|z|^{2}} \\
=\sum_{n=1}^{\infty} v_{n} \sup _{y>0} \sum_{j=1}^{n-1} \int_{\psi\left(\Omega_{j}\right)} \frac{d x}{|\psi(x+i y)|^{2}} .
\end{aligned}
$$

On the other hand, the sum on the right-hand side of (35) becomes

$$
\begin{aligned}
\sum_{n=1}^{\infty} v_{n} \sup _{y>0} & \left(\int_{\Omega_{n}} \frac{d m \psi_{y}^{-1}(z)}{\left|z-\gamma_{n}\right|^{2}}+\sum_{j=n+1}^{\infty} \int_{\Omega_{j}} \frac{v_{n}}{\left|\gamma_{n}\right|^{2}} d m \psi_{y}^{-1}(z)\right) \\
\simeq & \sum_{n=1}^{\infty} v_{n} \sup _{y>0} \int_{\psi\left(\Omega_{n}\right)} \frac{d x}{\left|\psi(x+i y)-\gamma_{n}\right|^{2}} \\
& +\sum_{n=1}^{\infty} \frac{v_{n}}{\left|\gamma_{n}\right|^{2}} \sup _{y>0} \sum_{j=n+1}^{\infty} m\left(\psi\left(\Omega_{j}\right)\right),
\end{aligned}
$$

where $m\left(\psi\left(\Omega_{j}\right)\right)=m\left(\left\{\psi(x+i y): x+i y \in \Omega_{j}\right\}\right)$. From the above series of estimates, we see that $\left\|C_{\psi}\right\|_{\mathcal{S}_{2}}$ is finite if and only if the sums in (36) and (37) are finite and from which our assertion follows.

\section{Conflict of Interests}

The author declares that there is no conflict of interests regarding the publication of this paper.

\section{References}

[1] C. Pommerenke, "Schlichte Funktionen und analytische Funktionen von beschränkter mittlerer Oszillation," Commentarii Mathematici Helvetici, vol. 52, no. 4, pp. 591-602, 1977.

[2] A. Aleman and J. A. Cima, "An integral operator on $H^{p}$ and Hardy's inequality," Journal d'Analyse Mathématique, vol. 85, pp. 157-176, 2001.

[3] A. Aleman and A. G. Siskakis, "An integral operator on $H^{P}$," Complex Variables, Theory and Application, vol. 28, no. 2, pp. 149-158, 1995.

[4] A. Aleman and A. G. Siskakis, "Integration operators on Bergman spaces," Indiana University Mathematics Journal, vol. 46, no. 2, pp. 337-356, 1997.

[5] A. Aleksandrov, "A simple proof of the Volberg-Treil theorem on the embedding of coinvariantsubspaces of the shift operator," Zap. Nauchn. Sem. S.-Peterburg. Otdel. Mat. Inst. Steklov., vol. 217, pp. 26-35, 1994.

[6] A. B. Aleksandrov, "On embedding theorems for coinvariant subspaces of the shift operator. II," Journal of Mathematical Sciences, vol. 110, no. 5, pp. 2907-2929, 2002.

[7] W. S. Cohn, "Carleson measures and operators on star-invariant subspaces," Journal of Operator Theory, vol. 15, no. 1, pp. 181-202, 1986. 
[8] B. Cohn, "Carleson measures for functions orthogonal to invariant subspaces," Pacific Journal of Mathematics, vol. 103, no. 2, pp. 347-364, 1982.

[9] W. S. Cohn, "Radial imbedding theorems for invariant subspaces," Complex Variables: Theory and Application, vol. 17, no. 1-2, pp. 33-42, 1991.

[10] S. Treil and A. Volberg, "Embedding theorems for invariant subspaces of the inverse shift operator," Journal of Mathematical Sciences, vol. 42, pp. 1562-1572, 1988.

[11] Y. Belov, T. Y. Mengestie, and K. Seip, "Discrete Hilbert transforms on sparse sequences," Proceedings of the London Mathematical Society. Third Series, vol. 103, no. 1, pp. 73-105, 2011.

[12] A. Borichev and Y. Lyubarskii, "Riesz bases of reproducing kernels in Fock-type spaces," Journal of the Institute of Mathematics of Jussieu, vol. 9, no. 3, pp. 449-461, 2010.

[13] Y. Belov, T. Y. Mengestie, and K. Seip, "Unitary discrete Hilbert transforms," Journal d'Analyse Mathématique, vol. 112, pp. 383393, 2010.

[14] T. Mengestie, "Compact Carleson measures from sparse sequences," The Rocky Mountain Journal of Mathematics, vol. 44, no. 1, pp. 223-234, 2014.

[15] J. E. Littlewood, "On inequalities in the theory of functions," Proceedings of the London Mathematical Society, vol. s2-23, no. 1, pp. 481-519, 1925.

[16] H. Schwartz, Composition operators on $H^{p}$ [Thesis], University of Toledo, Toledo, Ohio, USA, 1968.

[17] J. H. Shapiro, "The essential norm of a composition operator," Annals of Mathematics: Second Series, vol. 125, no. 2, pp. 375404, 1987.

[18] V. Matache, "Composition operators on Hardy spaces of a halfplane," Proceedings of the American Mathematical Society, vol. 127, no. 5, pp. 1483-1491, 1999.

[19] Y. Lyubarskii and E. Malinnikova, "Composition operator on model spaces," http://arxiv.org/abs/1205.5172.

[20] A. Aleman, Y. Lyubarskii, E. Malinnikova, and K.-M. Perfekt, "Trace ideal criteria for embeddings and composition operators on model spaces," http://arxiv.org/abs/1307.2652.

[21] T. Mengestie, Two weight discrete Hilbert transforms and systems of reproducing kernels [Ph.D. thesis], Norwegian University of Science and Technology, 2011.

[22] E. A. Nordgren, "Composition operators on hilbert spaces," in Hilbert Space Operators, vol. 693 of Lecture Notes in Mathematics, pp. 37-63, Springer, Berlin, Germany, 1978.

[23] P. Halmos, Measure Theory, Van Nostrand, Princeton, NJ, USA, 1950. 


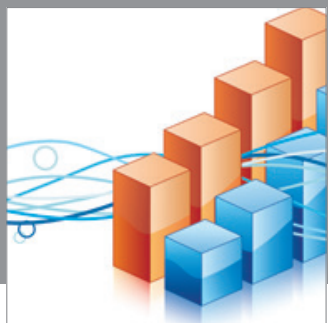

Advances in

Operations Research

mansans

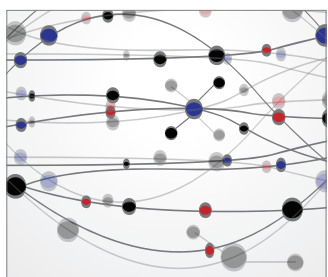

The Scientific World Journal
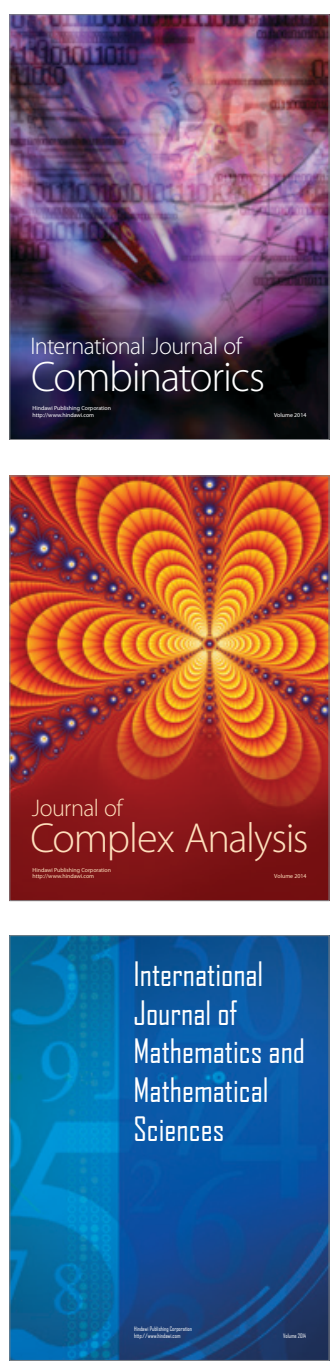
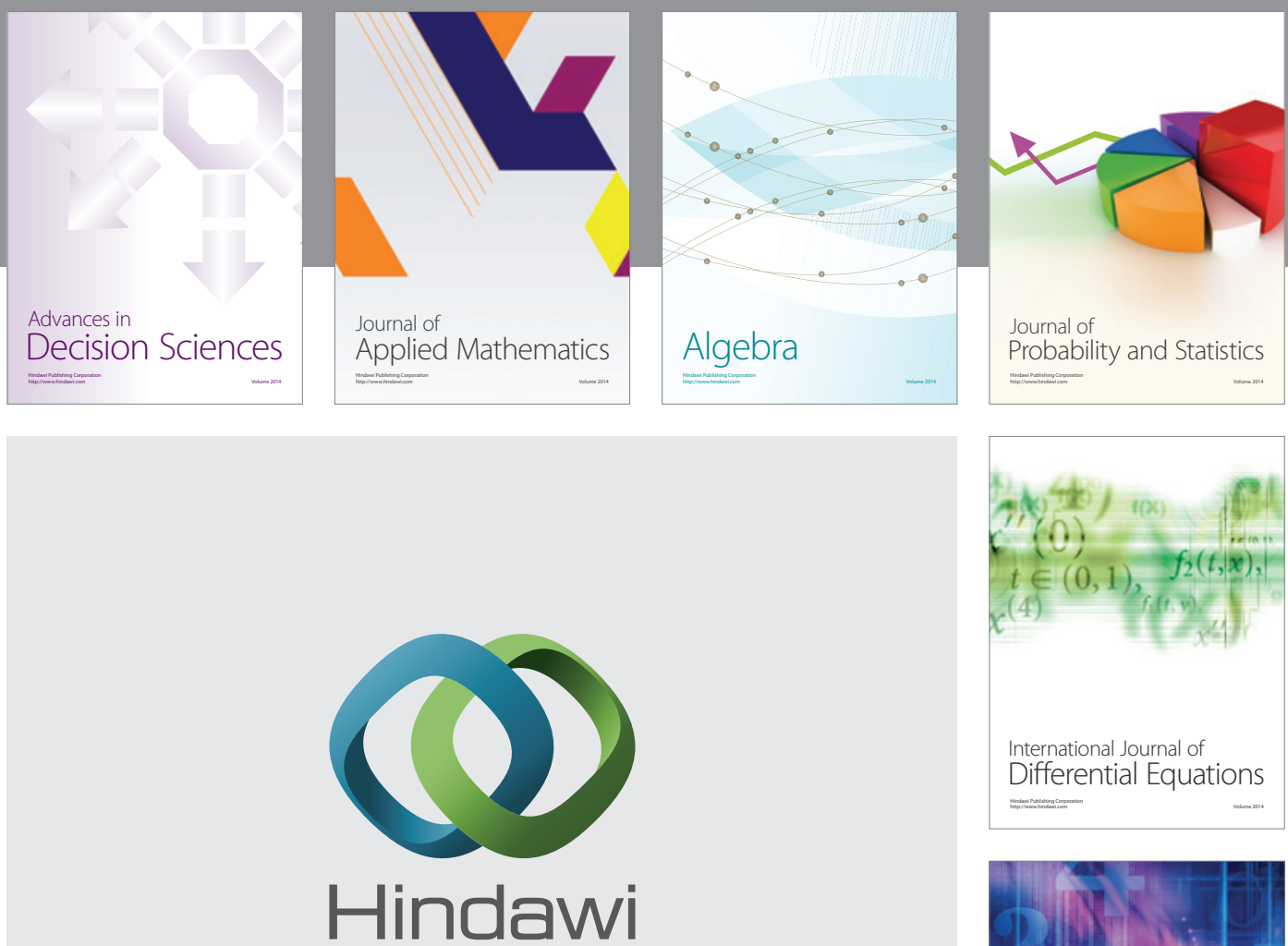

Submit your manuscripts at http://www.hindawi.com
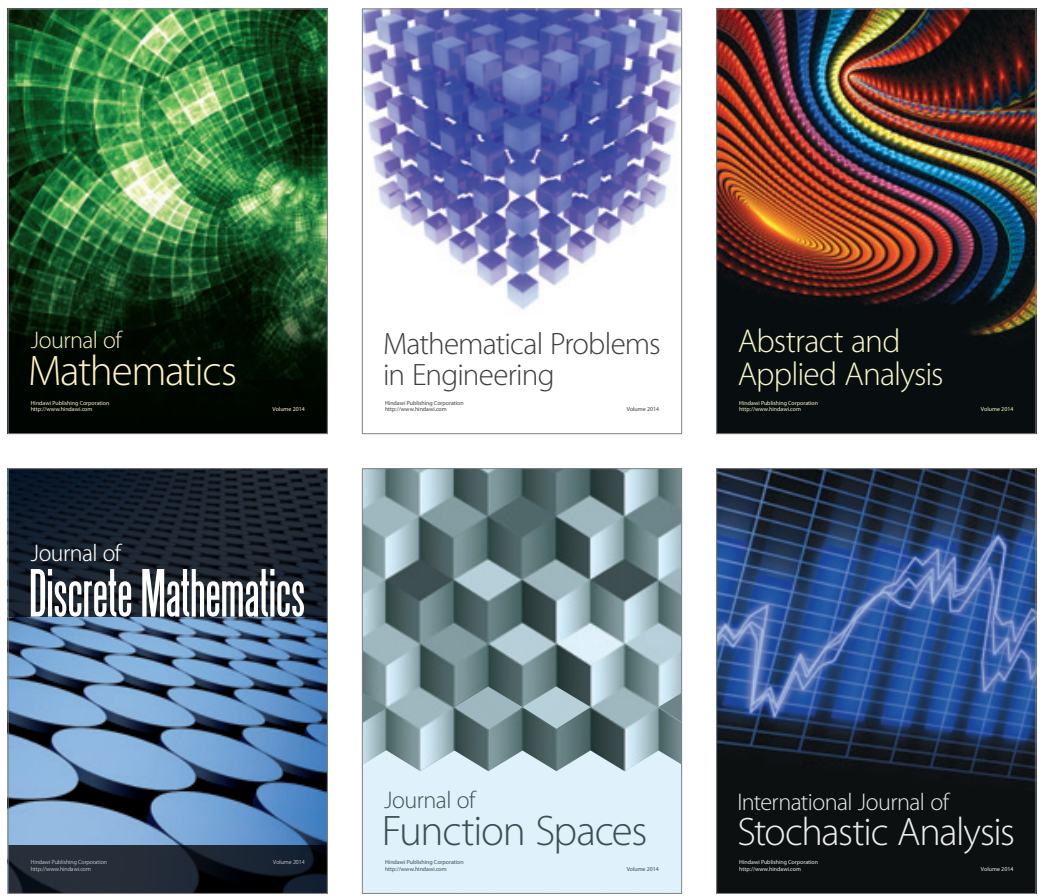

Journal of

Function Spaces

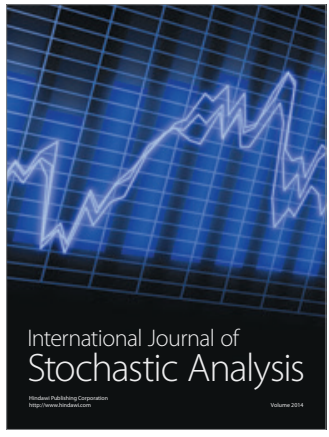

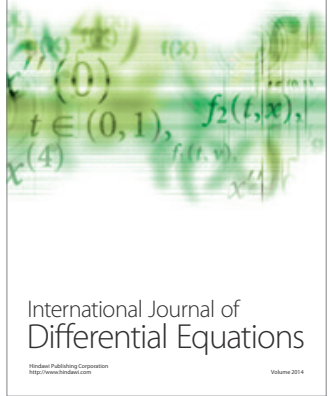
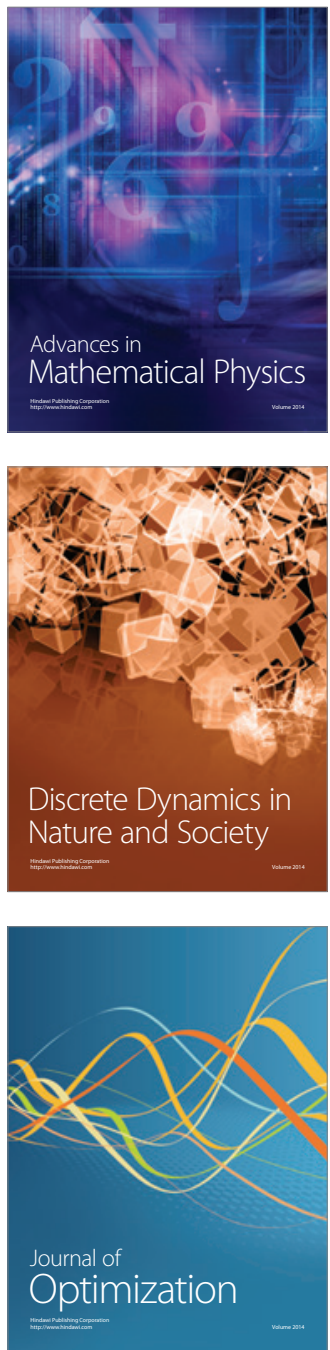